\title{
Design and Fabrication of a Frequency and Polarization Reconfigurable Microwave Antenna on a Printed Partially Magnetized Ferrite Substrate
}

\author{
Farhan. A. Ghaffar, Mohammad Vaseem, Langis Roy and Atif Shamim, Senior Member IEEE
}

\begin{abstract}
Design of a polarization reconfigurable and frequency tunable patch antenna operating in the partially magnetized state of a ferrite substrate is presented in this paper. A low cost additive manufacturing process based on a custom iron-oxide magnetic ink (to realize the magnetic substrate) and a silver-organo-complex metallic ink (to realize the antenna) has been used to demonstrate a novel fully printed magnetically controlled antenna. The magnetic substrate is characterized for its magnetostatic and microwave properties. The ink shows a saturation magnetic flux density of $156 \mathrm{mT}$ with a squareness of 0.26 . A prototype patch antenna at $6 \mathrm{GHz}$ is implemented on the magnetic substrate using inkjet printing. When excited by an external magnetic field the antenna shows frequency splitting and polarization reconfigurability. The measured results agree well with the simulation model of the antenna in the partially magnetized state. A maximum tuning range of $16.7 \%$ and $5 \%$ for the two splitted frequency points is obtained with circular polarizations of opposite sense. The results show the viability of reconfigurable and tunable $\mathrm{RF}$ components on partially magnetized substrates through low cost additive manufacturing techniques.
\end{abstract}

Index Terms - ferrite, magnetic materials, resonator, tunable devices, antenna, reconfigurability.

\section{INTRODUCTION}

Polarization reconfigurable, frequency tunable and wideband antennas are useful in various applications such as satellite communication, imaging and sensing [1]-[4]. There is a growing interest in using radio frequency (RF) components that can sense the available spectral environment and then reconfigure the system intelligently so as to provide efficient and secure communication. One major application in this regard is Software Defined Radios (SDR) [5]. This application requires antennas and other RF components to be reconfigurable so that they can be tuned to different frequencies depending on the availability of the frequency channels in the spectrum. It also requires antennas with polarization diversity in order to have secure communication while reducing the multipath effect. This means that antennas that are frequency and polarization reconfigurable are highly desirable for such applications.

Frequency tuning or reconfigurability of antennas can be achieved by various methods. One such method for controlling the performance of antennas is by employing magnetic materials such as ferrites. Ferrites have been classically used as substrates for tunable antennas, phase shifters and phase scanning arrays due to the ability to control the RF components realized on them through a variable magnetostatic bias [6]-[8]. A number of papers have been previously published on the theory and design of reconfigurable antennas using traditional ferrite substrates [9]-[12]. However, these antennas are designed to operate in the saturated state of the ferrite substrates. The authors have previously reported several tunable and reconfigurable microwave passive components on the partially magnetized ferrite Low Temperature Co-fired Ceramic (LTCC) substrate [13]-[15]. Although, the theory and measurements of these microwave components show the viability of operating the ferrite substrates in the unsaturated state, the cost of the ferrite LTCC technology is significant. Similarly, a polarization reconfigurable Ferrite Resonator Antenna (FRA) implemented on a partially magnetized substrate was reported in [16]. However, the theory of this design is different from a patch antenna

\footnotetext{
Manuscript received ....

Farhan. A. Ghaffar and Langis Roy are with Faulty of Electrical and Applied Science at University of Ontario Institute of Technology (UOIT), Canada

Mohammad Vaseem and Atif Shamim are with CEMSE Division of King Abdullah University of Science and Technology, Saudi Arabia
}

which is expected because of the design configuration. The results shown in [16] demonstrate the frequency splitting and circular polarization but do not establish the polarization sense of the two frequency points to be opposite. Furthermore, the authors did not measure the axial ratio or the polarization ratio of the antenna for different bias states which is important to understand the antenna operation at different magnetization levels. To cater to this, a low cost novel additive manufacturing process with custom magnetic and metallic inks [17], for the design of a reconfigurable circular patch antenna, is presented in this paper. The magnetic ink is initially characterized for its low frequency and high frequency properties. It is shown through simulations and measurements that when a DC magnetic field (normal to the substrate) is applied to a circular patch antenna, its center frequency with linear polarization splits into two different frequencies with circular polarizations of opposite sense of rotation. Through magnetostatic simulations, it is observed that the antenna is indeed operated in the partially magnetized state. The results indicate that the partially magnetized state can be used for the design of reconfigurable antennas by employing low cost additive manufacturing processes.

\section{PRINTING OF MAGNETIC INK AND ITS ChaRACTERIZATION}

Before undertaking the antenna design, it is important to explain the fabrication process and the subsequent material characterization, as the final stack up and the material properties are required for antenna design and simulations. Custom inks namely, magnetic ink made of iron-oxide nano-particles for substrate printing and a metallic ink made of silver-organo-complex (SOC) for patch antenna printing, have been developed in-house [18]. The magnetic ink is prepared by mixing iron oxide $\left(\mathrm{Fe}_{3} \mathrm{O}_{4}\right)$ nanoparticles in $\mathrm{SU}-8$ epoxy resin. Iron oxide nanoparticles with an average particle size of $20 \mathrm{~nm}$ were synthesized using a simple solution process [19], [20]. The as-synthesized iron oxide nanoparticles are chemically functionalized with oleic acid so as to be compatible with UV-curable epoxy resist. In the next step, the functionalized iron oxide nano-particles are mixed with the SU8 2000 (Microchem) epoxy resist in 50:50 weight \% ratio for formulating ink paste, as shown in Fig. 1 (a). Fig. 1 (b) and (c) shows the scanning electron microscopic (SEM) image and energy-dispersive spectroscopic (EDS) spectrum taken from the SU8-mixed iron oxide NPs. The SEM image (b) confirmed that the nanoparticles are almost spherical in shape and well embedded in the SU8 matrix. The EDS spectrum (c) demonstrates that the iron oxide nanoparticles embedded SU8 matrix is made of $\mathrm{Fe}, \mathrm{O}$ and $\mathrm{C}$. The high content of carbon is primarily due to SU8 molecule. Once the ink paste is ready, it is also characterized for its viscosity measurement which is showing the viscosity of $37.8 \mathrm{cP}$, using a spindle speed of $100 \mathrm{rpm}$ and shear rate of $132 \mathrm{~s}^{-1}$ at room temperature. The as-prepared ink can be printed using a manual screen printing technique, such as squeegee. The steps for the fabrication process are displayed in Fig. 2. An FR-4 board with a sacrificial paper on the backside is used as a support material in this work, however any other material can also be used instead of FR-4. This is done because initially the ink is in liquid form and needs a support substrate until it solidifies after curing (heating process to remove the solvent). A slot is first created in the support material using LPKF4 Protomat S103 (Fig. 2 (a)) to facilitate the printing of the magnetic ink for a precise substrate thickness of $1.5 \mathrm{~mm}$. For solidifying purposes, the ink is cured using an ultraviolet (UV) source for 30 min, as shown in Fig. 2 (b). Once the ink is solidified, it can be 
separated from the support material by cutting it from the edges as shown in Fig. 2 (c). The sacrificial paper on the back of the magnetic substrate is removed by immersing it in warm water for $10 \mathrm{~min}$. A 10 $\mu \mathrm{m}$ smoothening layer of ' $3 \mathrm{D}$ vero black plus' material is then inkjet printed and photo-cured on top of the magnetic substrate. In the next step, the SOC ink is deposited on top and bottom of the substrate using inkjet printing. A total of 8 layers of SOC ink are printed and cured using infrared (IR) heating for $5 \mathrm{~min}$. Both sides of the magnetic substrate are printed with the smoothening layer and the SOC ink as shown in Fig. 2 (d).
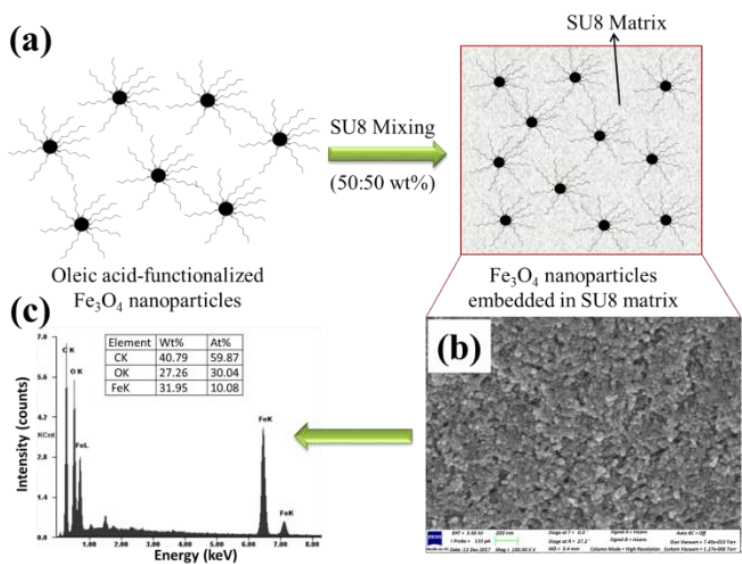

Fig. 1. (a) Functionalization of iron-oxide nanoparticles and mixing with SU8, (b) scanning electron microscopic (SEM) image of embedded nanoparticles in SU8 matrix and its (c) elemental analysis

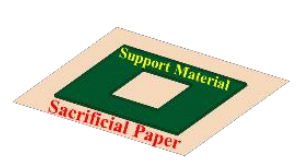

(a)

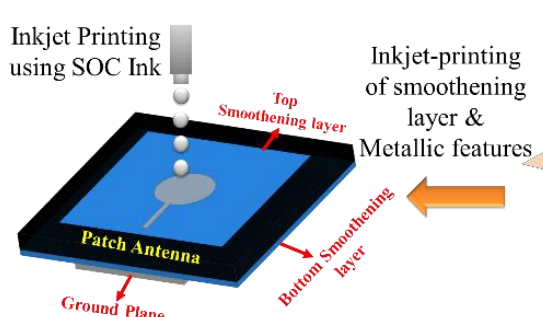

(d)

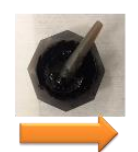

Screen printing of creen printing of

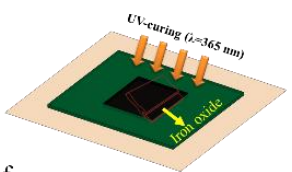

(b) Removing the substrate from FR4 screen

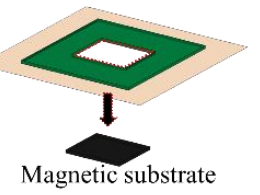

(c)
Fig. 2. Fabrication process for the magnetic substrate (a) FR4 support with backside sacrificial paper (b) Screen printing of iron oxide ink (c) After curing, cutting the solid magnetic substrate (d) Printing of smoothening layer and SOC layer on both sides of the substrate

It is important for the antenna design that the newly printed magnetic substrate be characterized for its material properties. The characterization of the ink is discussed in the following sections.

\section{A. Magnetostatic Characterization}

Vibrating Sample Magnetometer (VSM) is used for the hysteresis loop and $B(\mathrm{H})$ curve measurements of the magnetic ink. For this characterization, the substrate without any metallic layers on it is placed in the VSM. For the printed substrate, the measured results are displayed in Fig. 3. The magnetic ink demonstrates a saturation flux density $\left(B_{\mathrm{s}}\right)$, coercive field $\left(H_{\mathrm{c}}\right)$ and remanent flux density $\left(B_{\mathrm{m}}\right)$ of 156 $\mathrm{mT}, 3.66 \mathrm{kA} / \mathrm{m}$ and $35 \mathrm{mT}$ respectively. For tunable designs, greater the saturation magnetization, greater is the tunability of the component. For this work, the value of $156 \mathrm{mT}$ is acceptable and can provide decent tuning. However, for the future work, this value can be further increased by modifying the composition of the ink. From the saturation magnetization, the magnetization frequency of the material $\left(f_{\mathrm{m}}=2.8 \times 10^{10} \cdot \mu_{0} M_{\mathrm{s}}=4.37 \mathrm{GHz}\right)$ is calculated. Magnetization frequency is important as the ferrites do not show any low-field losses after this frequency [9]. It is recommended that the center frequency of a microwave device should be higher than the magnetization frequency of the medium to avoid any low field losses [21]. In order to estimate the loss of the ink, it is characterized for its linewidth $(\Delta H=100$ Oe at $3 \mathrm{GHz}$ ) using a half wavelength micrsotrip line resonator fabricated on Duroid 5880 board [22]. Another important characterization is related to the high frequency properties of the material. Therefore, once the material has been characterized for its magnetostatic properties, and its low loss region is known, a suitable resonant frequency can be selected for the resonator design. This resonator can be used to extract the microwave characteristics of the ink.

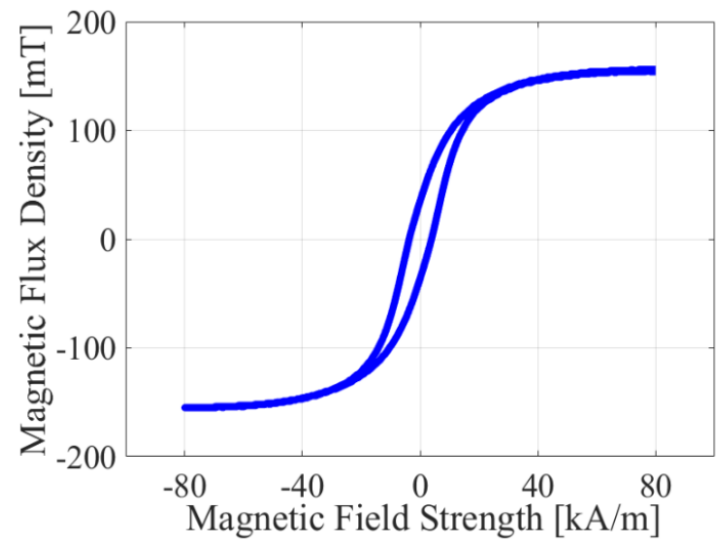

Fig. 3. Measured $B(H)$ curve of printed $\mathrm{Fe}_{3} \mathrm{O}_{4}$ (magnetic) ink

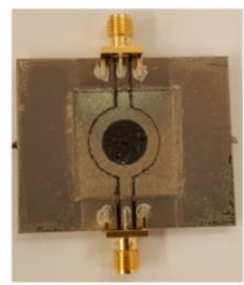

(a)

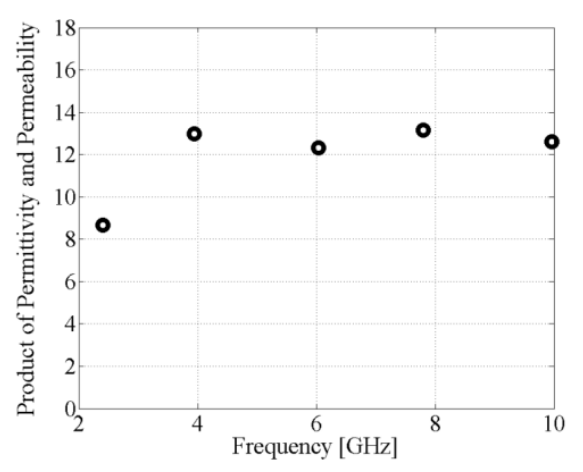

(b)
Fig. 4 (a) Fabricated ring resonator (b) Product of permittivity and permeability of the magnetic ink

\section{B. Microwave Characterization}

A coplanar waveguide (CPW) based ring resonator is fabricated on top of the printed magnetic substrate, as shown in Fig. 4 (a). The resonator has been inkjet printed on the magnetic substrate using the silver ink. The ring is designed with a radius of $6.75 \mathrm{~mm}$, having a conductor width and gap of $2.5 \mathrm{~mm}$ and $200 \mu \mathrm{m}$ respectively. Keeping in mind the typical dielectric constant of the ferrite materials $\left(\varepsilon_{\text {eff. }} \sim\right.$ 14), the dimensions of the ring are appropriate for a resonant frequency of $2 \mathrm{GHz}$. Although $2 \mathrm{GHz}$ is less than $f_{\mathrm{m}}$ of the substrate, it can still be used to extract the properties at the fundamental frequency (i.e. 2 $\mathrm{GHz}$ ) as well as for the higher order harmonics. In this way, the authors 
can confirm the higher loss at frequencies below $f_{\mathrm{m}}$ and lower loss at frequencies above $f_{\mathrm{m}}$.

These results have been used to extract the dielectric constant of the material. Since this is a magnetic material, the result obtained from the resonator will give a product of the initial permeability and permittivity. This product is displayed in Fig. 4 (b). As can be seen, the value of this product varies with respect to the frequency, which is expected due to the varying initial permeability of the ferrite material [19]. The demagnetized permeability of a magnetic material is given by (1) [23] and can be used here to isolate the two quantities. Using this equation, the permittivity and permeability of the medium at different frequencies are listed in Table I. Frequencies above $f_{\mathrm{m}}$ are considered in the table since these are the frequencies that can be used for the antenna design. For the initial design of any microwave device this product can directly be used in the equation of the resonant frequency. In addition to the dielectric constant, the loss tangent $(\tan \delta)$ of the material is also calculated from the measured results. The conductor losses are calculated using the transmission line calculator of Agilent ADS. The measured conductivity of the metal is $5 \times 10^{6}$ $\mathrm{S} / \mathrm{m}$, which is used to evaluate the conductor loss for different frequencies. Once the conductor loss is known, the dielectric loss of the material can be evaluated. The loss tangent of the substrate at frequencies below $f_{\mathrm{m}}$ is relatively higher. For example, at $2.4 \mathrm{GHz}$ and $4 \mathrm{GHz}$, the loss tangent values are 0.13 and 0.015 respectively. This is due to the low field losses of the magnetic material in the absence of the magnetic bias [21]. However, the loss tangent value at frequencies above $f_{\mathrm{m}}$ is in the acceptable range. For example, at $6 \mathrm{GHz}$, its value is 0.007. Therefore, $6 \mathrm{GHz}$ is used as the design frequency for the patch antenna design.

$$
\mu_{\mathrm{o}}^{\prime}=\frac{2}{3}\left[1-\left(\frac{f_{m}}{f_{\text {res. }}}\right)^{2}\right]^{\frac{1}{2}}+\frac{1}{3}
$$

Table I: Permittivity and permeability of the magnetic ink

\begin{tabular}{|c|c|c|}
\hline $\begin{array}{c}\text { Frequency } \\
{[\mathrm{GHz}]}\end{array}$ & $\begin{array}{c}\text { Demagnetized } \\
\text { Permeability } \\
\left(\mu^{\prime}{ }_{0}\right)\end{array}$ & $\begin{array}{c}\text { Permittivity } \\
\left(\varepsilon_{\text {eff }}\right)\end{array}$ \\
\hline 6.04 & 0.796 & 15.5 \\
\hline 7.9 & 0.86 & 15.29 \\
\hline 9.9 & 0.9 & 14.2 \\
\hline
\end{tabular}

\section{Design and Simulations of Patch Antenna}

The equation for the resonant frequency of a circular patch antenna on a normally biased saturated ferrite substrate is given by [9]:

$$
J_{1}^{\prime}(k a) \pm \frac{\kappa}{a \mu} J_{1}(k a)=0
$$

where $J_{1}$ is the Bessel function of first kind, $k$ is the wavenumber, $\mu$ and $\kappa$ are the elements of the permeability tensor [9] and $a$ is the radius of the circular patch antenna. Here, $k$ should be evaluated using the equation derived in [9]. At a given magnetization inside the substrate $\mu$ and $\kappa$ can be calculated using the equations for the partially magnetized state [13]. Using the antenna dimension, $a$ one can solve for the values of $k$ using (2). These roots obtained from (2) give the frequency points where the antenna operates and can be compared with the simulations and measurements. For the design and simulations of the antenna, the substrate properties obtained in the last section are used. The simulations comprise of two parts i.e., magnetostatic and microwave simulations. The magnetostatic simulations have been carried out in CST EM Studio, while CST Microwave studio is used for the high frequency simulations. For the magnetostatic simulations a magnet has been used as a source to generate the bias inside the substrate. Varying the applied magnetic field from the electromagnet varies the internal magnetic field inside the substrate and the magnetization. Once the magnetization inside the substrate is known it can be used to evaluate the elements of the permeability tensor [13]. This permeability tensor of the substrate can be defined in a microwave simulator to study the performance of the antenna in the partially magnetized state.

\section{A. Magnetostatic Simulations}

For any electromagnet, the applied magnetic field is known and is defined by the user. This value of the applied field can be defined in the magnetostatic simulator to record the strength of the magnetization $\left(\mu_{0} M\right)$ inside the substrate. The external field applied to a magnetic substrate reduces by the time it reaches inside the substrate due to the loss at the air-to-ferrite interface (also known as demagnetization effect). As a result, the magnetic field applied to a substrate is different from the internal field generated inside the substrate. To record the values of the internal magnetic field, the strength of the field is observed at the center of the substrate. To record a single value of the magnetic field inside the substrate, three lines, one along each axis (i.e. $\mathrm{X}, \mathrm{Y}$ and $\mathrm{Z}$ ), are used on which the fields are observed. The dimensions of the three lines are equal to the size of the substrate, which is $24 \mathrm{~mm} \times 24 \mathrm{~mm} \times 1.5 \mathrm{~mm}$ respectively. This strategy is similar to the one used by the authors in the previous reported ferrite based microwave designs [13]-[15]. The field strengths obtained from the three lines are averaged in order to generate a single value for the internal magnetization inside the substrate. The results obtained from these simulations are shown in Fig. 5. As the applied magnetic field is increased, both the internal magnetic field and the magnetization inside the substrate increases, as expected. For a magnetic field strength of $25.1 \mathrm{kA} / \mathrm{m}$, the internal magnetization $\left(\mu_{0} M\right)$ is recorded at approximately $133 \mathrm{mT}$, which means that the material is almost saturated. To maintain the operation in the partially magnetized state, this is the highest value of the magnetic field used in the simulations.

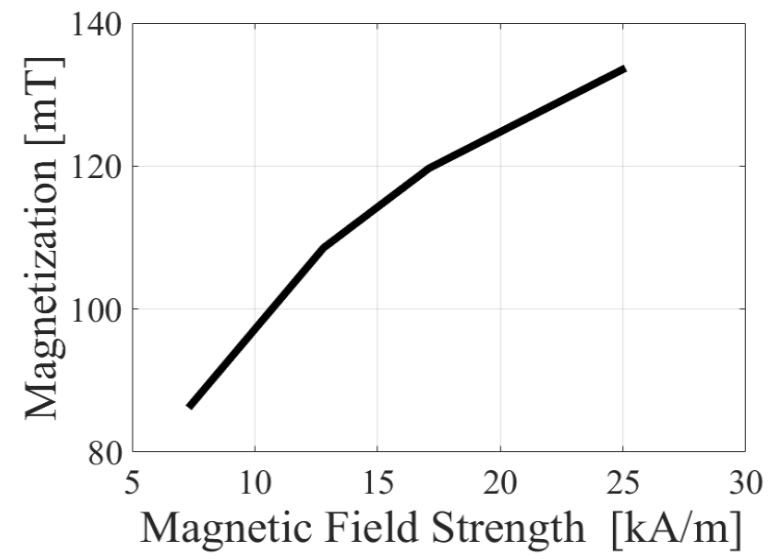

Fig. 5. Magnetization inside the substrate vs the magnetic field

\section{B. Microwave Simulations}

Initially, the circular patch antenna is designed on the magnetic substrate without any bias. The antenna is optimized to operate at 6 $\mathrm{GHz}$ using the dielectric and magnetic properties of the material. These properties include the dielectric constant, the demagnetized permeability and the loss tangent obtained from section III. The optimized antenna has a radius of $4.1 \mathrm{~mm}$ on a substrate with thickness of $1.5 \mathrm{~mm}$. The antenna is fed at the edge with a microstrip feed therefore it needs to be matched using an open circuit stub. The open circuit stub has a length of $3.5 \mathrm{~mm}$ and is $1.6 \mathrm{~mm}$ away from the edge of the antenna. The inset feeding technique is not suitable here because of the circular polarization of the antenna. Therefore, instead of using inset feed, a conventional microstrip stub matching is used in this 
design. The simulated impedance and the radiation performance of the antenna are presented in Fig. 6. A maximum gain of $3.0 \mathrm{dBi}$ is obtained from the antenna at $6 \mathrm{GHz}$. The antenna shows a good match at $6 \mathrm{GHz}$ with the maximum radiation in the bore-sight as expected. Once the antenna has been optimized for its performance in the unbiased state, it can be simulated for the partially magnetized state.

The antenna is simulated with a uniform bias inside the substrate changing the magnetization of the antenna from 0 to the saturation magnetization $\left(\mu_{0} M_{\mathrm{S}}\right)$ value of $156 \mathrm{mT}$. The simulation results show the frequency splitting of the antenna. As expected in the biased state [9], the antenna resonates at two different frequencies with opposite sense of polarization. This means that a linearly polarized antenna starts behaving as a circularly polarized antenna. In simulations, one polarization tunes from $6 \mathrm{GHz}$ to $6.4 \mathrm{GHz}$, while the other polarization tunes from $6 \mathrm{GHz}$ to $5 \mathrm{GHz}$. If the direction of the applied bias is reversed, then the senses of polarization change for the two tuning frequencies, which means that if the $6 \mathrm{GHz}$ to $6.4 \mathrm{GHz}$ band was showing LHCP for one direction of bias, then it will show RHCP for the opposite bias direction and vice versa. The actual comparison between the simulations will be discussed in detail with the measurements in the following section.

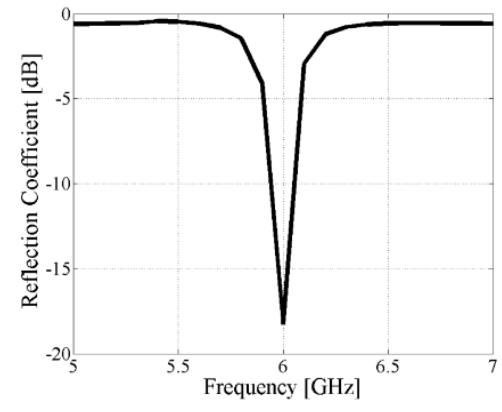

(a)

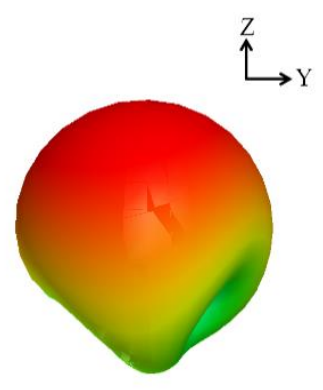

(b)
Fig. 6. Simulated results of circular patch antenna (a) Reflection coefficient (b) $3 \mathrm{D}$ radiation pattern

\section{Measurements of the Patch Antenna}

The photograph of the fabricated antenna is shown in the inset of Fig. 7. An SMA connector is mounted on the antenna through a conductive silver epoxy (CW-2460 of Chemtronics) for its impedance and radiation pattern characterization. As expected, the antenna works at $6 \mathrm{GHz}$ in the unbiased state, as shown in Fig. 7. Some loss can be seen in the impedance plot of the antenna as the curve for the reflection coefficient does not go close to $0 \mathrm{~dB}$. This is most likely due to the large surface roughness of the magnetic substrate and subsequently the conductor layer. For this device, the value of the roughness is measured to be $4.5 \mu \mathrm{m}$ which causes significant loss in the antenna radiated power. The conductor layer printed on the substrate is only $2 \mu \mathrm{m}$ thick which means that the conductor layer also experiences the roughness due to the underneath substrate. The loss observed in the measured reflection coefficient of the antenna can also be seen in the gain measurements, which will be discussed in the gain and radiation pattern measurements section.

\section{A. Impedance Measurements in the biased state}

For characterization under biased condition, the antenna is placed between the poles of the electromagnet whose magnetic field is varied to test the antenna under varying bias conditions. The antenna is placed in a way that the applied direction of bias is normal to the

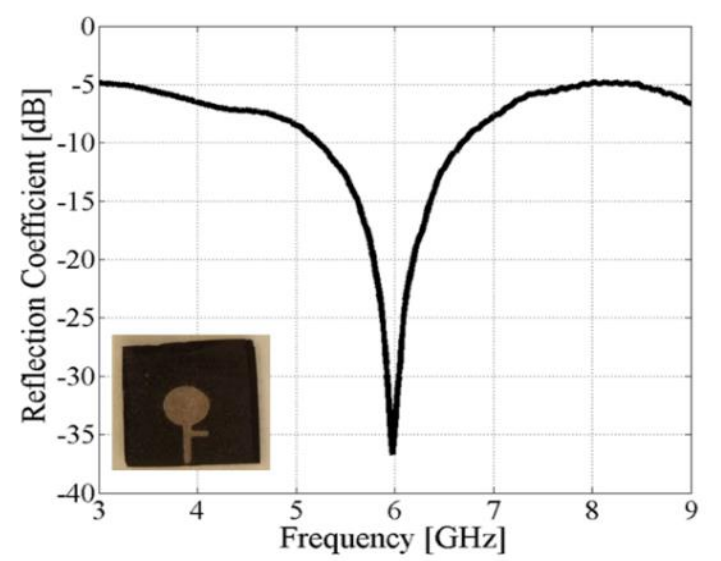

Fig. 7. Measured reflection coefficient of the antenna with an image of fabricated module add coordinates

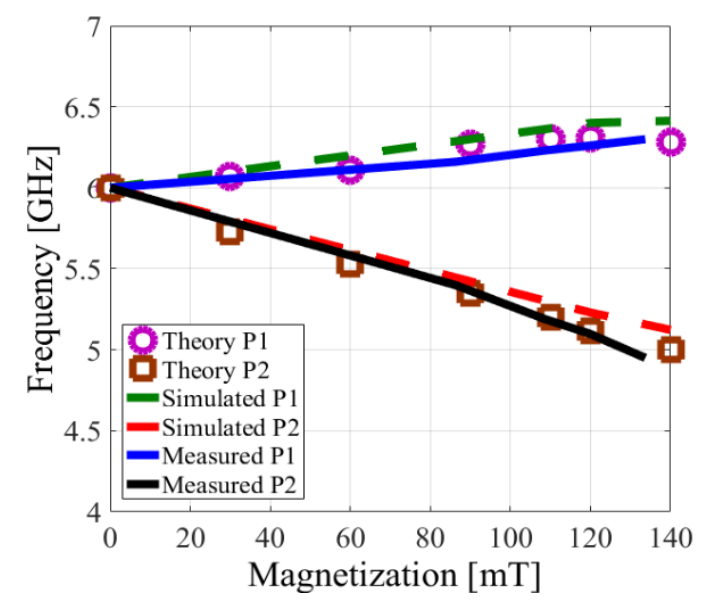

Fig. 8. Frequency splitting of the circular patch antenna due to the applied magnetostatic bias in theory, simulations and measurements

surface of the antenna. As has been seen in simulations, biasing the antenna causes a split in the resonance frequency of the antenna. As the strength of the magnetic bias is increased the two frequencies tune away from each other. A plot showing the comparison between the theory (using (2)), simulations and measurements is shown in Fig. 8. A good agreement can be observed between these results which validates the operation of the antenna in the unsaturated state. The two new resonant frequencies maintain good impedance matching for different bias strengths. From the simulations, it is known that the two frequencies have circular polarization with opposite sense of rotation. One of the polarizations is referred to as P1 while the other one is named as P2. Each polarization (P1 or P2) can either be LHCP or RHCP depending on the direction of the bias. For $+\mathrm{Z}$ direction of bias $\mathrm{P} 1$ is observed to be LHCP while P2 is found to be RHCP. If the direction of bias is reversed to $-\mathrm{Z}$ direction, then the two polarizations will interchange.

Although the phenomenon of frequency splitting has been observed in the impedance measurements, the polarization is yet to be determined for the fabricated antenna. This means that the antenna needs to be characterized for its gain and radiation performance in the biased conditions.

\section{B. Gain and Radiation Pattern Measurements}

The radiation pattern of the antenna is initially measured in the unbiased state at $6 \mathrm{GHz}$. A good agreement can be seen between the simulated and measured radiation performance from Fig. 9. The 
antenna shows linear polarization in the unbiased state as expected from the simulations with cross polarization levels of $15 \mathrm{~dB}$. A slight tilt is observed in the measured radiation pattern of the antenna in Phi $=0^{\circ}$ plane. This is the plane where the connector of the antenna is mounted due to which the maximum gain of the antenna is at $\theta=15^{\circ}$, instead of $\theta=0^{\circ}$. This is consistent with the previous reported work on tunable antennas where the size of the antenna is actually smaller than the connector [13], [14].

For the biased state radiation pattern measurement, a permanent magnet is used with the antenna inside the anechoic chamber. The radiation patterns are measured at $5.6 \mathrm{GHz}$ and $6.1 \mathrm{GHz}$ for the two frequency points, shown in Fig. 10. The two radiation patterns show circular polarization with the axial ratio of $6 \mathrm{~dB}$. The reason for a poor value of axial ratio will be explained in the next section. The maximum measured gain of the antenna is found to be $-1 \mathrm{dBi}$. This value is low as compared to the simulated value of $3.0 \mathrm{dBi}$. As discussed before, this low gain performance from the antenna is due to the high surface roughness value $(4.5 \mu \mathrm{m})$ measured for the substrate and the conductor [14], [15]. In order to verify this effect, the measured surface roughness value is used in the simulator for the post-measurement simulations. These simulations show that the gain of the antenna drops from $3.3 \mathrm{dBi}$ to $-1 \mathrm{dBi}$ due to this effect. A plot showing the gain of the antenna against the increasing conductor surface roughness is shown in Fig. 11. It can be seen that the gain of the antenna keeps decreasing due to the increase in the surface roughness of the conductor for a constant thickness. This means that optimizing the fabrication process for better surface roughness value will improve the radiation performance considerably.

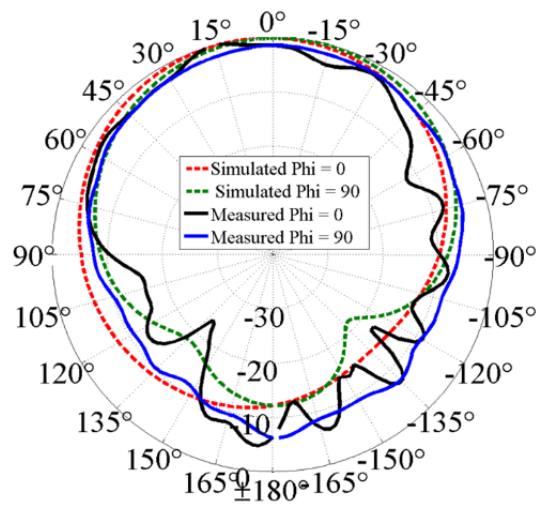

Fig. 9. Simulated and measured radiation patterns in the unbiased state

\section{Axial Ratio and Antenna Polarization Measurements}

Ideally the complete radiation characteristics of the antenna should be measured in an anechoic chamber with the required magnetic source. However, it is not possible to accomplish this task due to the use of electromagnet as the source for generating the required strong magnetostatic fields. Using the electromagnet inside our chamber is not practically feasible, due to its size which is in the order of $60 \mathrm{~cm} \mathrm{x}$ $50 \mathrm{~cm} \times 40 \mathrm{~cm}$, as shown in Fig. 12 (a). Instead of an electromagnet, a permanent bar magnet can be used in the chamber, as shown in Fig. 12 (b). The size of this magnet ( $50 \mathrm{~mm} \times 1 \mathrm{~mm} \times 1 \mathrm{~mm}$ ) is suitable for the radiation pattern measurements inside the chamber but such a magnet cannot generate strong enough magnetic fields to assess the polarization splitting. Also, using a single magnet at the base of the antenna will not ensure magnetic fields that are perfectly normal to the substrate. Therefore, to further verify the proposed simulations a measurement set up has been devised. A non-magnetic receiving antenna is used on one port of the vector network analyzer (VNA) while the transmitting port is connected to the magnetic antenna under test. The transmission coefficient $S_{21}$ is then measured for the horizontal and vertical polarizations of the receive antenna by rotating

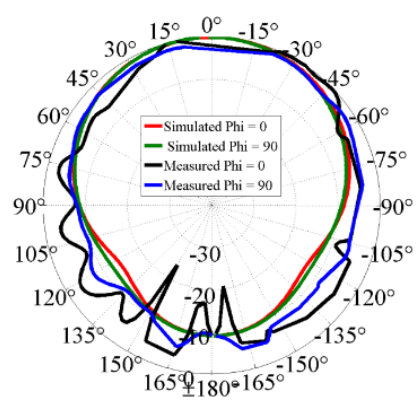

(a)

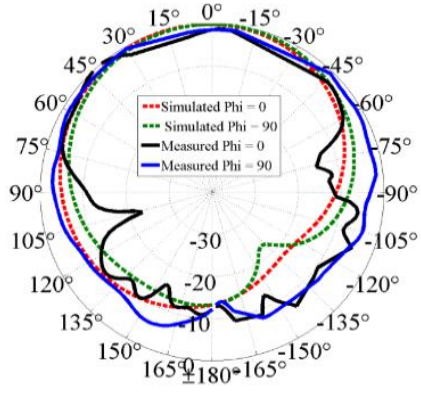

(b)
Fig. 10. Measured radiation patterns in the presence of a magnet at (a) $5.6 \mathrm{GHz}$ (b) $6.1 \mathrm{GHz}$

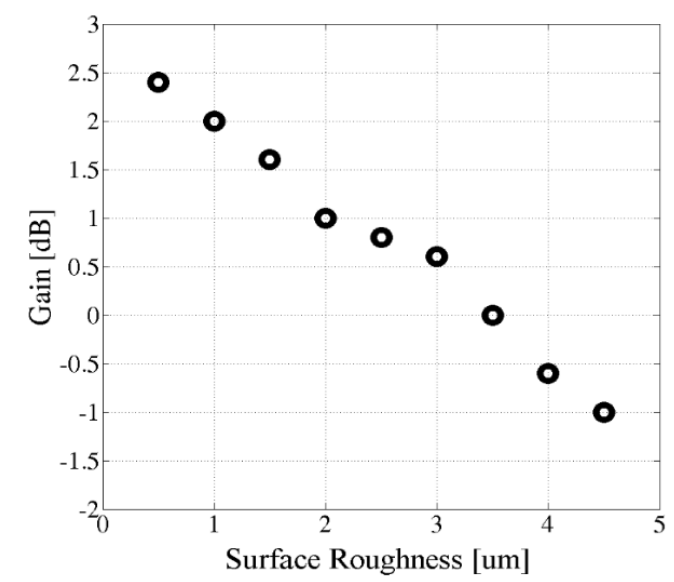

Fig. 11. Gain vs surface roughness of the antenna conductor

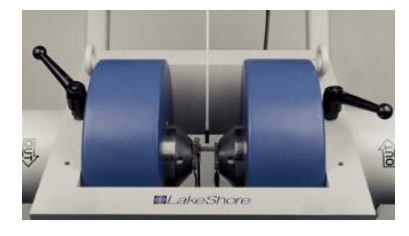

(a)

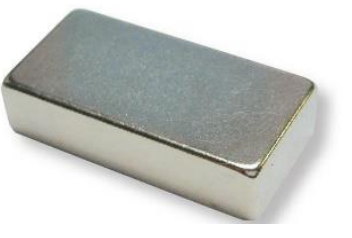

(b)
Fig. 12 (a) Electromagnet and (b) Permanent magnet used for biasing of antenna [24], [25]

it $90^{\circ}$ for each measurement, as shown in Fig. 13. This is done for different values of the applied magnetic field. The ratio of the two perpendicular field components gives the axial ratio at a particular frequency. Using the frequencies from Fig. 8, the axial ratio is measured and is shown in Fig. 14. The general trend of this graph is that the axial ratio becomes lower with the applied magnetic field. This trend is also consistent with the simulations. This is because as the ferrite medium approaches saturation, more magnetic dipoles align with the applied magnetic bias fields. At saturation, all the dipoles are aligned with the magnetic field providing a fixed axis for the RF magnetic field to rotate hence providing better circular polarization [9]-[12]. However, the slight variation in the AR value between the two polarizations can be due to the environment in which the antenna is measured. Since the antenna is placed in between the poles of the electromagnets, it is bound to have some reflections. Nonetheless, the measured results still validate the circular polarization of the antenna in the biased state for two different frequencies.

Although the axial ratio measurements do verify the antenna to be circularly polarized, it does not verify the sense of the polarization. To determine this, RHCP and LHCP non-magnetic antennas have been fabricated at $5.2 \mathrm{GHz}$ and $6.3 \mathrm{GHz}$. These antennas are used as the 
receive antennas just as the linearly polarized antenna has been used in the previous measurements (Fig. 11). Using the two antennas with the opposite sense of polarization can help in deciding the sense of the polarization of the antenna under test. At $5.2 \mathrm{GHz}$, it is observed that the value of $S_{21}$ is $19 \mathrm{~dB}$ greater for the case when RHCP antenna is used as the receive antenna as compared to the scenario when LHCP antenna is used. This means that at $5.2 \mathrm{GHz}$ the magnetic antenna shows an LHCP polarization. Similar measurements are performed at $6.3 \mathrm{GHz}$, where it is observed that the LHCP receive antenna shows a $14 \mathrm{~dB}$ greater $\mathrm{S}_{21}$ as compared to the RHCP receive antenna. These measurements prove that the magnetic antenna is RHCP at the measured frequency which verifies the theory and simulations.

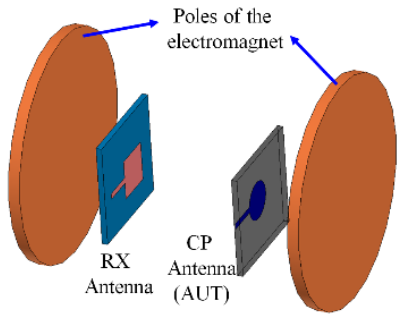

(a)

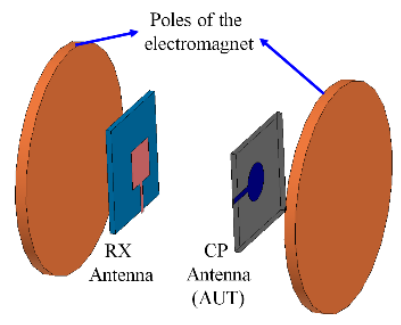

(b)
Fig. 13. Test set up for AR measurement of the CP antenna (a) RX antenna with horizontal polarization (b) RX antenna with vertical polarization

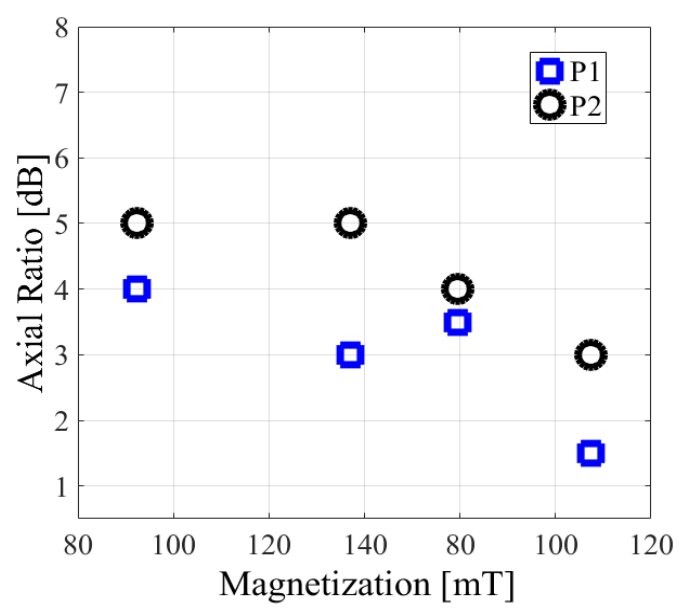

Fig. 14. Measured axial ratio vs. the magnetization

\section{CONCLUSION}

The paper presents the design of a polarization reconfigurable and frequency tunable circular patch antenna on a partially magnetized substrate. A custom iron-oxide nano-particle based magnetic ink is used to print the magnetic substrate and a silver ink is used to print the antenna. The ink is initially studied for its magnetostatic and microwave properties. Once the characteristics of the ink are known, a circular patch antenna is realized using it. Full wave simulations of the antenna are carried out which show the phenomenon of frequency splitting and polarization reconfigurability. The prototype antenna is then fabricated and measured for its impedance and radiation performance. The antenna shows a measured tuning range of $5 \%$ and $16.7 \%$ for the two polarizations. A good correlation is observed between the theory, simulations and the measurements. Since the fully printed process has been used for the first time, there are some short comings, such as large surface roughness. However, with further optimization of the process this problem can be overcome and efficient tunable antennas can thus be realized. The future demonstrations using this magnetic ink can show it as a substitute to the multilayer ferrite LTCC technology by employing embedded bias windings.

\section{REFERENCES}

[1] C. G. Christodoulou, et. al., "Reconfigurable Antennas for Wireless and Space Applications," Proc. of IEEE, vol. 100, no. 7, pp. 2250-2261, 2012.

[2] W. Lin and H. Wong, "Wideband Circular Polarization Reconfigurable Antenna," in IEEE Trans. Ant. Prop., vol. 63, no. 12, pp. 5938-44, 2015.

[3] F. A. Ghaffar A. Shamim "Design of silicon based fractal antennas" Microwave and Optical Technology Letters (MOTL), vol. 55, no. 1, pp. 180-186, 2013.

[4] F. A. Ghaffar, A. Shamim, and K. N. Salama, "Design and comparison of LTCC based fractal antennas," in Proc. URSI General Assembly Sci. Symp., 2011.

[5] O. Hiari, R. Mesleh, "A Reconfigurable SDR Transmitter Platform Architecture for Space Modulation MIMO Techniques," in IEEE Access, vol. 5, pp. 24214-24228, 2017.

[6] D. M. Pozar, V. Sanchez, "Magnetic tuning of a microstrip antenna on a ferrite substrate", Elect. Lett., vol. 24, no. 12, pp. 729-731, 1988.

[7] A. Petosa, et. al., "Magnetically tunable ferrite resonator antenna", Elect. Lett., vol. 30, no. 13, pp. 1021-1022, 1994.

[8] A. Nafe, A. Shamim, "An Integrable SIW Phase Shifter in a Partially Magnetized Ferrite LTCC Package," in IEEE Transactions on Microwave Theory and Techniques, vol. 63, no. 7, pp. 2264-2274, 2015.

[9] D. M. Pozar, "Radiation and scattering characteristics of micrsotrip antennas on normally biased ferrite substrates," IEEE Trans. Ant. Prop., vol. 40, no. 9, pp. 1084-1092, 1992.

[10] A. Petosa, et. al., "Switchable LP/CP ferrite disk resonator antenna," Elect. Lett., vol. 31, no. 3, pp. 148-149, 1995.

[11] G. Leon, et. al., "Characteristics of aperture coupled microstrip antennas on magnetized ferrite substrates," in IEEE Transactions on Antennas and Propagation, vol. 53, no. 6, pp. 1957-1966, 2005.

[12] K. K. Tsang, R. J. Langley, "Design of circular patch antennas on ferrite substrates," IEE Proc. Micro. Ant. Prop., vol. 145, no. 1, pp. 49-55, 1998.

[13] F. A. Ghaffar, et. al., "Theory and design of a tunable antenna on a partially magnetized ferrite LTCC substrate", IEEE Trans. Ant. Prop., vol. 62, no. 3, pp. 1238-1245, 2014.

[14] F. A. Ghaffar, Atif Shamim, "A Ferrite LTCC based dual purpose helical antenna providing bias for tunability," IEEE Ant. Wless Prop. Lett., vol. 14, pp. 831-834, 2015.

[15] F. A. Ghaffar, A. Shamim, "A Partially magnetized ferrite LTCC based SIW phase shifter for phased array applications", IEEE Trans. Mag., vol.51, no.6, pp.1-8, 2015.

[16] T.-W. Kim, et. al., "A theoretical model for frequency response of a cylindrical resonator antenna with the $\mathrm{HE}^{11} \delta$-Mode splitting behavior," IEEE Trans. Ant. Prop., vol. 65, no. 4, pp. 1615-1623, 2017.

[17] G. McKerricher, et. al., "Fully inkjet printed RF inductors \& capacitors using polymer dielectric \& silver conductive ink with through vias", IEEE Trans. Elect. Dev., vol. 62, no. 3, pp.1002-1009, 2015.

[18] M. Vaseem, et. al., "Robust design of a particle-free SOC ink with high conductivity \& inkjet stability for flexible electronics", ACS App. Mat. Intfc., vol. 8, no. 1 pp. 177-186, 2016.

[19] F. A. Ghaffar, et. al., "A ferrite nano-particles based fully printed process for tunable microwave components," IEEE MTT-S Intl. Micro. Symp. (IMS), pp. 1-3, 2016.

[20] F. A. Ghaffar, et. al., "A fully printed ferrite nano-particle ink based tunable antenna," IEEE Intl. Symp. Ant. \& Prop. (APS/URSI), pp. 10591060, 2016.

[21] A. Shamim, et. al., "Ferrite LTCC based antennas for tunable SoP applications," IEEE Trans. Comp. Packag. Manu. Tech., vol. 1, no. 7, pp. 999-1006, 2011.

[22] A. S. Sokolov, "Broadband ferromagnetic resonance linewidth measurement by a microstripline transmission resonator", Applied Physics Letters, vol. 108, no. 17, 2016.

[23] E. Schlomann, "Microwave behavior of partially magnetized ferrites", Journal of Applied Physics, vol. 41, no. 1, pp. 204-214, 1970.

[24] Lakeshore 7400 series electromagnet: http://www.lakeshore.com/products/Vibrating-SampleMagnetometer/7400-Series-VSM/Pages/Overview.aspx

[25] Quader magnet: https://www.magno-sphere.de/quadermagnete-neodym-10-x-5-x$1 \mathrm{~mm} / \mathrm{a}-1025 /$ 